\title{
AFFECTIVE COMPUTING AND COLLABORATIVE NETWORKS: TOWARDS EMOTION-AWARE INTERACTION
}

\author{
Andrej Luneski \\ South-East European Research Centre, Thessaloniki, GREECE, anluneski@seerc.org \\ Roger K. Moore \\ The University of Sheffield, UK, r.k.moore@dcs.shef.ac.uk
}

\begin{abstract}
Affective computing is a young field of research that interconnects many different domains and has a range of important applications. However, little attention has been given to the employment of affective computing in collaborative networks. This paper examines the possible challenges for 'injecting' emotion-awareness into collaborative networks with a view to improving the effectiveness, motivation and naturalness in the communication between their members. Moreover, by observing the state of the art in affective computing, we present certain needs and benefits (why) as well as possible approaches (how) to the application of affective computing technologies in collaborative networks, with the focus on affective interaction.
\end{abstract}

\section{INTRODUCTION}

The constant and rapid evolution of information and communication technologies has enabled efficient and effective collaboration among geographically dispersed networks of people. In particular, recent years have witnessed the appearance of a range of virtual communities, virtual organizations, virtual enterprises and other types of 'collaborative networks' (CNs) (Camarinha-Matos, 2005). Since its introduction as a new scientific discipline, $\mathrm{CN}$ is facing many challenges primarily focused on the improvement of the collaboration process. Apart from efficiency, both the business and the scientific communities desire naturalness in their communications.

Nowadays, intelligent, natural and effective interaction is considered to be one that incorporates the recognition and expression of emotion, i.e. affective interaction (Salovey, 1990). However, emotions were formerly considered as an exceptional human ability that gets in the way of effective and intelligent human communication and is undesirable for rational behaviour (Goleman, 1995). As a result, emotions have only recently entered into the spectrum of attractive and significant research topics. A principal reason for this is a growing appreciation of the strong interconnection between human emotions and intelligence (Salovey, 1990, Goleman, 1995). Indeed, the realisation of the significance of emotions in the expression of intelligence gave birth to the term 'emotional intelligence', defined as "the capacity to understand emotional information and to reason with emotions" (Salovey, 1990).

Affective computing (AC) is the scientific discipline that is concerned with emotional interactions performed with and through computers (Picard, 1997). Picard 
(1997) defines AC as "computing that relates to, arises from, or deliberately influences emotions". In fact, AC is an interdisciplinary field that introduces new domains and applications mostly, but not exclusively, related to advanced humancomputer interaction (HCI). Affective HCI assumes that the computer is emotionaware, i.e. it is both able to recognise emotions and to intelligently or appropriately express them. In the investigation of possible application areas of AC, researchers have recently been interested in virtual environments, communities and networks, among others (ref), although not much has been achieved so far.

This paper discusses the main challenges arising from the application of AC technologies in the sphere of collaborative networks. By presenting the key aspects and the state-of-the-art in $\mathrm{AC}$, we examine possible future directions and fields where emotion-aware and emotionally-intelligent technologies might provide particular gains. Moreover, the paper investigates the need (why) of affective interaction in $\mathrm{CN}$, the benefits arising from 'injecting' emotions into the communication channels and the possible approaches (how) towards the application of affective computing technologies in collaborative networks, with focus on affective interaction.

\section{NEED FOR AFFECTIVE INTERACTION IN CN}

Emoticons have been used as a tool for the 'graphical' representation of the emotion hidden behind a textual message, and their extensive usage (whether in a form of a simple smile or a 3D cartoon avatar) provides clear evidence that people feel the need to convey their emotions to the other party in their communications. Therefore, it is argued that the nature of human expression requires an 'injection' of emotions into the communication in $\mathrm{CN}$ platforms.

In order to understand what $\mathrm{CN}$ might gain from affective interaction ${ }^{1}$, we first have to examine the impact of emotions on some key aspects of human forms of communication and collaboration. In particular, emotions directly influence human physical and especially mental states and this in turn has an impact on the performance in $\mathrm{CN}$. The very principal underlying any type of $\mathrm{CN}$ is the improvement of communication within networks where people with mutual goals are striving to come up with the best output collaboratively. The quality of the collaboration and the output might directly depend on the creativity of the members of a CN. According to the 'broaden-and-build model' (Fredrickson, 2003), positive emotions play a key role in increasing an individual's creativity. Moreover, emotions can control the motivation for achieving goals (Cooper et al, 2000; Parkinson 1995; Brewer, 2004) as well as improving a person's problem solving ability (Damasio, 1994). A person in a happy state is most likely to accomplish their pre-set goals, and will perform better than if they were in a negative emotional state such as anger or depression. Affective interaction could be used as a positive instrument to create emotional-awareness between the members of a CN.

\footnotetext{
${ }^{1}$ The term 'affective interaction' often refers to human-human interaction. When discussing $\mathrm{CN}$, affective interaction includes any computer-related interaction i.e. computer-mediated human-human, computer-generated or computer-interpreted interaction.
} 
Additionally, it can help in the discovery of methods for increasing the level of performance, creativity and motivation inside a $\mathrm{CN}$.

Many researchers consider empathy to be a strong mechanism for reducing frustration and generally improving the emotional state and mood (Hone, 2006; Klein et al., 2002). We argue here that empathy is one of the missing aspects that differentiate communication in CNs from direct physical human-human interaction. With affective interaction, users are capable of expressing their empathy towards other CN participants (empathic interaction), thereby increasing the closeness and the levels of trust between $\mathrm{CN}$ members.

The naturalness of the affective communication process brings about increased interactiveness and, by focusing on the $\mathrm{CN}$ participant's involvement, it sets the base for the sense of "social presence". Social presence was introduced by Shoer et al and is defined as "the salience of the other in a mediated communication and the consequent salience of their interpersonal interactions" (Shoer et al, 1976). The expression of emotion, feelings, and mood in a mediated interaction has been considered as a defining characteristic of social presence (Garrison et al., 2000). The notion of social presence in CNs can be realised with ubiquitous computing where the computer still acts as a mediator in the communication but it is demilitarised as a single entity i.e. the participants do not 'notice' the computer-mediation process. This issue is further discussed later in the paper.

Furthermore, a $\mathrm{CN}$ is described as a network of entities that are "geographically dispersed and "heterogeneous in terms of their...culture..." (Camarinha-Matos, 2006). There are many theories that support the notion of universal emotions, according to which emotions are expressed in the same manner, regardless of the cultural diversities (Ekman, 1972, Mesquita,1992, Plutchik, 2001). If these theories are adopted in the domain of $\mathrm{CN}$, emotional expression and interaction might bridge the potential cultural or even the social gap between the diverse entities in a $\mathrm{CN}$.

In summary, affective interaction, as a new and more advanced type of interaction, can bring about a certain level of excitement to $\mathrm{CN}$ participants, as well as, change the whole perception between them.

\section{EMOTION AWARENESS AND INTELLIGENT EMOTIONAL EXPRESSIVNESS IN A CN}

In affective communication there is a need for emotion-awareness and emotionally intelligent expressiveness. Awareness is known to play a crucial role in a collaborative environment i.e. people need to be 'aware' of each other in such an environment in terms of their intentions, general feelings, influence on the shared workspace etc. (Garcia et al, 1999). Since we are concerned with computer-mediated affective interaction, the computer ${ }^{2}$ has to posses the ability of emotion-awareness and through emotion expressiveness to enable users to be aware of the emotional state of others. In other words, it has to be aware of the emotional state of the user

\footnotetext{
${ }^{2}$ By computer we mean the communication mediator (it does not necessarily refer to the notion of a desktop computer)
} 
on one side of the communication (input), interpret, transmit and, on the other side, express it in an emotionally intelligent and effective manner.

Emotional input can be comprised of one, or preferably a combination of all, channels and from the different input forms a clear interpretation/specification of emotional state has to be obtained. Additionally, that emotion specification is expressed in one or a combination of emotional output channels. Therefore, when referring to emotion-awareness and emotional intelligence in CNs we are focusing on the computer means for exhibiting these capabilities. In order to do so, the computer has to be equipped with $\mathrm{AC}$ mechanisms and technologies.

\subsection{Emotion awareness}

The complexity of the emotion has been studied for a long time and researchers still debate on the theory(s) that most appropriately describes this 'human ability'. One group of researchers state that there exists a set of basic emotions or emotion categories, such as anger, fear, joy, sadness, disgust etc. (Plutchik, 1990; Ekman, 1971). Others use the 'dimensional approach' for describing affective space emotions, where emotions are represented through values in an n-dimensional space (Scholesberg, 1954; Lang et al, 1997). The affective space typically consists of two dimensions: arousal (calm/excited) and valence (negative/positive). Furthermore, there have been many studies and debates on the affective states that a person can experience and the channels through which those states are expressed to the outer world. For an affective communication in $\mathrm{CN}$ we are interested in the most proper communicative channels of emotion, through which the user can portray to the computer and through which the computer can identify the emotional state of the user. This is not exclusive to the output/expressive channels (since the internal physiological reactions to emotions are not necessarily visible by others). Mainly, emotions are communicated/expressed vocally, through facial expressions and through gestures (Clynes 1977; Planalp, 1999). Additionally, through specific sensors the computer can identify the emotions form physiological signals (Bauer, 1998).

Vocal Emotion Communication. Human speech caries emotional information in the semantics and in the speech prosody. While semantics (what has been said) is a more obvious expression of emotion, prosody holds more detailed emotional information. Prosody combines nonsemantic cues in spoken language, such as: fundamental frequency (pitch), loudness, rhythm, formant structure of speech sounds, intonation etc. An extensive research has been done on affect encoding and decoding in vocal communication (Banse, 1996; Cowie and Douglas-Cowie, 1996; Bachorowski 1999). See (Scherer, 2003) for more comprehensive review. Generally studies have been oriented in recognition of six basic emotions (anger, joy sadness, fear, disgust and surprise) from speech and significant results have been reached (Yu et al, 2001; Dealert et al, 1996; Nogueiras et al, 2001). The average success rate in the recognition process is between $70 \%$ and $80 \%$, which is higher that the human recognition rate of around $60 \%$. 


\section{$\underline{\text { Affective facial expressions and gestures }}$}

Facial expression are mostly used by humans in identifying certain emotional categories due to the distinct facial expressions for the "universal" human emotions such as happiness, anger, sadness, surprise, fear and disgust (Ekman, 1972).

Body gestures play a vital role in conveying human emotional states, and due to the high variability of the emotional body posture and gesture facial expression are strongly influenced by the concurrently presented body language (Ambady, 1992; Meeren, 2005).

Affective psychophysiology. Most of the disturbance caused by a certain emotion influences the internal physiology of the human body (alteration of brain states, increase of the heart-beat rate, hands sweating, (face) muscle movements, increase of the respiration rate etc). Monitoring these types of emotional expressions requires employment of specific sensors and recordings of their output such as Electroencephalogram (EEG), Electrocardiogram (ECG), Skin Conductnce or Electrodermal Activity (EDA), Electromyogram (EMG) etc. The usage of physiological signals in identifying a user's emotional state has became popular in recent years due to the advanced development and availability of unobtrusive sensors that can provide constant and reliable monitoring of a user's internal emotional reaction (Picard, 1997). Physiological sensors have been integrated into clothing and jewellery; skin conductivity sensor in shoes, blood volume pressure sensor in earrings, respiration sensor in a sports bra and numerous others (Picard, 2001). Bamidis et al have proposed a multi-channel framework for experimenting with physiological sensing of human emotion (Bamidis et al, 2007).

\subsection{Emotionally expressiveness}

Human-like and emotional intelligence is the key factor that is required in the computer for the user to feel that is engaged into a natural, intelligent and realistic communication. In a $\mathrm{CN}$, the computer acts as a representation of the person on the other side of the communication. As such, not only it has to provide the best possible interpretation of the other person, but it should provide additional information that may assist the user in connecting better with that representation as if it were the other person. Going along with this requirement is the belief of philosophers and psychologists that "what matters in human-human interaction is the individual's subjective belief about each other, not the objective truth of the interaction" (Bailenson et al, 2000). Computer appearance is one of the principal design issues for emotional expression; it has to provide the user with an interface similar to a face-to-face interaction (Picard, 1997).

Avatars have been used as a visual representation of human characters for more natural human-computer interaction. They are seen as "the physical representation of the self in virtual reality" (Castronova, 2003). Moreover, emotional interaction through avatars has already been seen in collaborative virtual environments and other types of collaborative systems (Fabri, 2005; Garcia, 1999; Selvarajah, 2005). Studies have shown that users interact with avatars as with other human beings (Bailenson, 2005). Since the intention of the avatar is to visually represent a human character, emotional expressiveness would require adoption of all the principles 
behind the human visual emotional expressiveness. More specifically, the relation between emotions and facial expressions, as well as, body gestures, as described in the previous section. Apart from the visual, emotions can be expressed through affective synthetic speech.

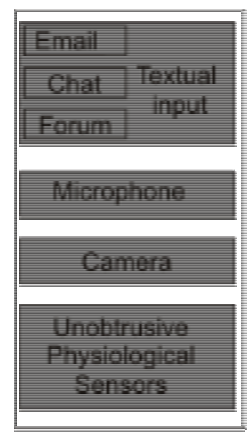

Emotion awareness 1NPUIT

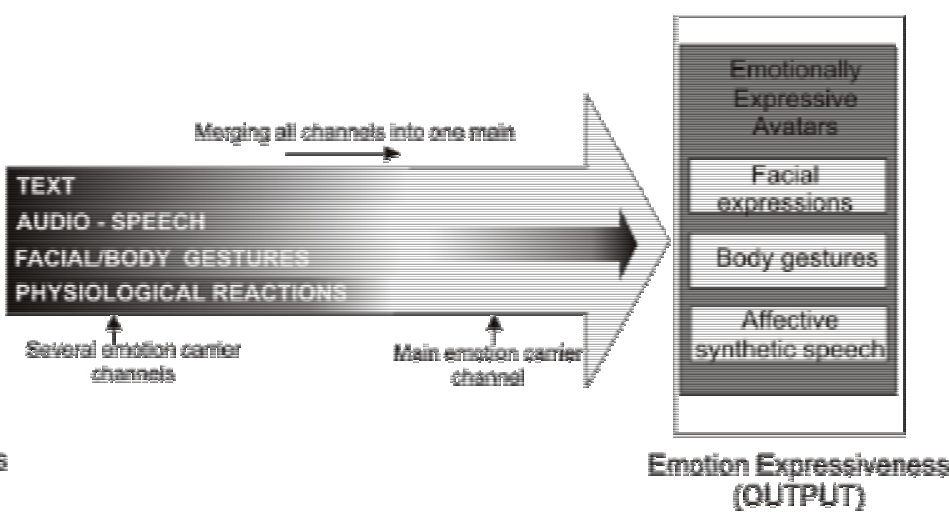

Figure 1 - Affective Interaction in $\mathrm{CN}$

\section{DISCUSSION}

The main aim of this paper was to review the needs and significance of emotions in human interaction, and the key aspects of affective interaction in $\mathrm{CN}$, based on the facts in the existing literature. We have discussed the impact of emotions on human rational behaviour in $\mathrm{CN}$, as environments with computer-mediated interaction human-human interaction. By presenting the key aspects of affective computing in relation to affective interaction we merely described possible application mechanism of affective computing in $\mathrm{CN}$. $\mathrm{CN}$ as an interdisciplinary topic includes many domains that can benefit from affective interaction and emotion-awareness among the participants. Imagine a scenario where tasks or projects have to be appointed to team/group members. If the delegator knows which team member is mostly excited or motivated for a specific task, a most effective delegation would be achieved and the team could have greater success. Moreover, decision support and group conflict mechanism can be constructed, based on emotional interaction, which could be used in electronic meetings inside CN (Garcia, 1999).

Collaborative Innovation Network (CoIN) is a type of $\mathrm{CN}$ defined as "a cyberteam of self-motivated people with a collective vision, enabled by the Web to collaborate in achieving a common goal by sharing ideas, information, and work" (Gloor, 2005). Affective interaction is specifically important for CoIN since it is a non-hierarchical $\mathrm{CN}$ with principles based on honesty, trust, innovation, creativity motivation, transparency and closeness. 


\section{REFERENCES}

Ambady N, Rosenthal, R. "Thin slices of expressive behaviour as predictors of interpersonal consequences: A meta-analysis". Psychological Bulletin, 1992; 111(2). Pp 256-274.

Bachorowski JA. "Vocal expression and perception of emotion". Current direct. Psychol. Sci., 1999; 8 (2), pp 53-57.

Bailenson J, Beall A, Blascovich J, Raimundo M, Weishbush M. "Intelligent agents who wear your face: User's reactions to the virtual self". Technical Report. Center for the Virtual Environment and Behaviors, Department of Psychology, University of California, Santa Barbara, 2000.

Bamidis PD, Luneski A, Vivas A, Papadelis C, Maglaveras N, Pappas C. "Multi-channel physiological sensing of human emotion: insights into Emotion-Aware Computing using Affective Protocols, Avatars and Emotion Specifications". In Proceedings of Medinfo 2007. Brisbane, Australia. pp 1068-72.

Banse R, Scherer KR. “Acoustic profiles in vocal emotion expression”. J. Pers. Soc. Psychol, 1996; 70 (3). Pp 614-636.

Burgoon JK et al. "Augmenting human identification of emotional states in video. In International Conference on Intelligent Data Analysis, 2005.

Bauer RM. "Physiologic Measures of Emotion". Journal of Clinical Neurophysiology: 15(5), 1998. pp 388-396.

Brewer MB, Hewstone M, Emotion and Motivation: Perspectives on Social Psychology, Blackwell Publishing, 2004.

Camarinha-Matos LM, Afsarmanesh H. "Collaborative Networks: A New Scientific Disipline", Journal of Intelligent Manufacturing: 16 (4-5),2005. pp 439-452, 2005.

Camarinha-Matos L, Afsarmanesh H, "Collaborative networks: Value creation in a knowledge society", In: PROLAMAT 2006, IFIP Int. Conf. On Knowledge Enterprise - New Challenges, Shanghai (CH), 2006.

Castronova E. "Theory of the Avatar". CESifo Working Paper Series No. 863, Fevruaty 2003.

Clynes M., "The Touch of Emotions",.Anchor Press/Double Day, 1977.

Cooper B, Brna P, Martins A. "Effective Affective in Intelligent Systems - Building on Evidence of Empathy in Teaching and Learning". in Ana Paiva (Ed.) Affective Interactions: Towards a New Generation of Computer Interfaces. Springer Verlag, 2000. pp 21-34.

Cowie R, Douglas-Cowie E. "Automatic statistical analysis of the signal and prosodic signs of emotion in speech”, In: Proc. ICSLP_96, Philadelphia, 1996. pp. 1989-1992.

Damasio A. Descarte's Error: Emotion, Reason and the Human Brain. Avon, New York, 1994.

Dellaert F, Polzin TS, Waibel A. "Recognizing emotion in speech". Int. Conf. on Spoken Language Processing (ICSLP), Pittsburgh, 1996.

Ekman, P. "Universals and cultural differences in facial expressions of emotion". In J. Cole (Ed.), Nebraska Symposium on Motivation 1971; 19. Lincoln, NE: University of Nebraska Press, 1972. pp 207-283.

Fabri M, Moore DJ. "The use of emotionally expressive avatars in Collaborative Virtual Environments". in Proceedings of the AISB Symposium on Virtual Social Agents. University of Hertfordshire, UK. April 2005. pp 88-95.

Fredrickson BL. The value of positive emotions. American Scientist. 2003: 91. pp 330-335.

Garcia O, Favela J, Machorro R. "Emotional Awareness in Collaborative Systems," spire, p. 296, String Processing and Information Retrieval Symposium \& International Workshop on Groupware, 1999.

Gloor P. Swarm Creativity: Competitive Advantage Through Collaborative Innovation Networks, 2005.

Garrison R, Anderson T, Archer W. Critical inquiry in a text-based environment: Computer conferencing in higher education, Unpublished manuscript, 2000.

Goleman D, Emotonal Intelligence, Bantham Books, New York, NY, 1995.

Hone K. "Empathic Agents to Reduce User Frustration: The Effects of Varying Agent Characteristics", Interacting with Computers, 2006; 18 (2), pp 227-245.

Kapoor A, Picard RW. "Multimodal affect recognition in learning environments". In ACM International Conference on Multimedia, 2005.

Klein J, Moon Y, Picard RW. "This computer responds to user frustration: theory, design and results". Interacting with Computers, vol. 14, issue 2, pp. 119-140, 2002

Lang, PJ, Bradley MM, Cuthbert BN. 1997). "Motivated attention: affect, activation and action". In Attention and orienting: sensory and motivational processes, P. J. Lang, R. F. Balaban ed. Hillsdale, NJ: Lawrence Erlbaum Associates, Inc, 1997. pp 97-134. 
Mesquita B, Frijda, NH. "Cultural variations in emotions: A review”. Psychological Bulletin; 112, 1992. pp 179-204.

Meeren H, Heijnsbergen C, Gelder B. "Rapid perceptual integration of facial expression

and emotional body language". Procedings of the National Academy of Sciences of USA, 2005; 102(45): pp 16518-16523.

Nogueiras A, Moreno A, Bonafonte A, Mariño JB. "Speech emotion recognition using hidden markov models". In Proceedings of Eurospeech, Aalborg, Denmark, 2001.

Parkinson B, Colman AM. Emotion and Motivation. Longman, London, UK, 1995.

Planalp S. Communicating Emotion: Social, Moral, and Cultural Processes (Studies in Emotion and Social Interaction). Cambridge University Press, 1999.

Plutchik R, Kellerman H. "A general Psychoevolutionary theory of Emotion, in Emotion Theory, Research, and Experience”. Academic Press, 1990; 1. pp 1980-1990.

Plutchik R. The nature of emotions. American Scientist, 2001; 89. pp 344, 2001.

Picard RW. "Affective Medicine: Technology with Emotional Intelligence". In Future of Health Technology, R. G. Bushko ed. OIS Press, 2001.

Ravindra De Silva P, Bianchi-Berthouze N. "Modeling human affective postures: an information theoretic characterizatino of posture features". Computer Animation and Virtual Worlds, 2004;15. pp 169-276.

Salovey P, Mayer JD. "Emotional Intelligence”, Imagination, Cognition and Personality 1990; 9. pp 185221.

Scholesberg H. "Three Dimensions of Emotions”, Psychological review, 1954.

Short J, Williams E, Christie B. The social psychology of telecommunications, Toronto, ON: Wiley, 1976.

Selvarajah K, Richards D. "The use of emotions to create believable agents in a virtual environment",.In Proceedings of the Fourth international Joint Conference on Autonomous Agents and Multiagent Systems-AAMAS'05, 2005.

Willyard CH, McCLees CW, “'Motorola’s Technology Roadmap Process”, Research Management , pp 13-19, Sept.-Oct. 1987.

Yu F, Chang E, Xu YO, Shum HY. "Emotion Detection From Speech To Enrich Multimedia Content", in the Second IEEE Pacific-Rim Conference on Multimedia, October 24-26, 2001, Beijing, China. 